\title{
RETRACTIONS AND HYPERSPACES
}

\author{
Sergio Macías \\ Universidad Nacional Autónoma de México, Mexico
}

\begin{abstract}
Retractions between hyperspaces have been studied by many authors. We study retractions form $m$-fold hyperspaces onto $n$-fold symmetric products.
\end{abstract}

\section{INTRODUCTION.}

Retractions between hyperspaces have been studied by many authors, for example see: $[13,14][8,15,25-27,29]$. In most cases retractions between $2^{X}$ and $\mathcal{C}(X)$ or $2^{X}$ and $\mathcal{F}_{1}(X)$ or $\mathcal{C}(X)$ and $\mathcal{F}_{1}(X)$ have been investigated. Very few results involving $2^{X}$ and $\mathcal{F}_{n}(X)$ or $\mathcal{C}_{n}(X)$ and $\mathcal{F}_{n}(X)$ exist $([25-27])$. Here we investigate the existence of a retraction from $\mathcal{C}_{m}(X)$ onto $\mathcal{F}_{n}(X)$, where $m$ and $n$ are positive integers such that $m \geq n$.

The paper is divided in four sections. After the definitions and notations, in section 3, we give some results that we use later, for example, we prove that if $X$ is a proper circle-like continuum, then its second symmetric product does not have trivial shape (Theorem 3.4). In section 4, we present our main results, for example, we give necessary conditions in order to have that $\mathcal{F}_{n}(X)$ is a retract of $\mathcal{C}_{m}(X)$ (Theorem 4.1). The arc is the only arc-like continuum $X$ for which there is a retraction from $\mathcal{C}_{m}(X)$ onto $\mathcal{F}_{n}(X)$ (Corollary 4.3). If $X$ is a one-dimensional continuum containing a simple closed curve, then there does not exist a retraction from $\mathcal{C}_{m}(X)$ onto $\mathcal{F}_{n}(X)$ (Theorem 4.5). If $\mathcal{F}_{n}(X)$ is an absolute retract, then $\mathcal{F}_{n}(X)$ is a strong deformation retract of $\mathcal{C}_{m}(X)$ (Theorem 4.9). For a continuum $X$, the existence of a retraction

2010 Mathematics Subject Classification. 54B20.

Key words and phrases. Absolute retract, arc-like continuum, arcwise decomposable continuum, circle-like continuum, continuum, decomposable continuum, indecomposable continuum, movable continuum, $n$-fold hyperspace, retraction, trivial shape, uniformly pathwise connected, uniquely arcwise connected, weakly chainable continuum. 
$R: \mathcal{C}_{m}(X) \rightarrow \mathcal{C}_{n}(X)$ such that $A \subset R(A)$ for all $A \in \mathcal{C}_{m}(X)$ is equivalent to the fact that $X$ is locally connected (Theorem 4.16).

\section{Definitions And nOtation.}

Given a subset $A$ of a metric space $Z$ with metric $d$, we denote by $C l(A)$ the closure of $A$. Also, $\mathcal{V}_{r}(A)$ denotes the open ball of radius $r$ about $A$.

A map is a continuous function. Let $f: X \rightarrow Z$ be a surjective map between metric spaces and let $\varepsilon>0$. Then $f$ is an $\varepsilon$-map provided that $\operatorname{diam}\left(f^{-1}(z)\right)<\varepsilon$ for all $z \in Z$. Let $Z$ be a metric space and let $A$ be a closed subset of $Z$. A retraction from $Z$ onto $A$ is a map $r: Z \rightarrow A$ such that $r(a)=a$ for all $a \in A$. The set $A$ is called a retract of $Z$.

Let $Z$ be a metric space. By a deformation we mean a map $H: Z \times$ $[0,1] \rightarrow Z$ such that $H((z, 0))=z$. Let $A=\{H((z, 1)) \mid z \in Z\}$. If the map $r: Z \rightarrow A$ given by $r(z)=H((z, 1))$ is a retraction from $Z$ onto $A$, then $H$ is a deformation retraction from $Z$ onto $A$. If $H$ is a deformation retraction from $Z$ onto $A$ and for each $a \in A$ and each $t \in[0,1], H((a, t))=a$, then $H$ is a strong deformation retraction from $Z$ onto $A$. The set $A$ is called a deformation retract of $Z$ (strong deformation retract of $Z$, respectively). A metric space $Z$ is an absolute retract provided that for each embedding $e: Z \rightarrow X$ of $Z$ into a metric space $X$ such that $e(Z)$ is closed in $X, e(Z)$ is a retract of $X$.

A compact metric space $Z$ lying in the Hilbert cube $\mathcal{Q}$ is movable if for every neighborhood $U$ of $Z$ in $\mathcal{Q}$, there exists a neighborhood $U_{0}$ of $Z$ contained in $U$ which is deformable inside $U$ into any neighborhood of $Z$.

A continuum is a nonempty compact connected metric space. A continuum $X$ has trivial shape provided that each map from $X$ into an absolute neighborhood retract is homotopic to a constant map. A tree is a continuum which can be written as a finite union of arcs, any two of which are either disjoint or intersect at only one of their end points and not containing a simple closed curve.

A continuum $X$ is arc-like (circle-like or tree-like) provided that for each $\varepsilon>0$, there exists an $\varepsilon$-map $f: X \rightarrow[0,1]\left(f: X \rightarrow \mathcal{S}^{1}\right.$, where $\mathcal{S}^{1}$ is the unit circle or $f: X \rightarrow Y$, where $Y$ is a tree). A proper circle-like continuum is a circle-like continuum which is not an arc-like continuum. The continuum $X$ is weakly chainable if it is a continuous image of an arc-like continuum.

A continuum $X$ is unicoherent provided that every time $X$ is the union of two subcontinua $A$ and $B$, we have that $A \cap B$ is connected. The continuum $X$ is hereditarily unicoherent if each of its subcontinua is unicoherent. An arcwise connected continuum is arcwise decomposable if there exist two proper arcwise connected subcontinua $A$ and $B$ of $X$ such that $X=A \cup B$. An arcwise connected continuum $X$ is uniquely arcwice connected if $X$ does not 
contain simple closed curves. A dendroid is an arcwise connected hereditarily unicoherent continuum. A dendrite is a locally connected dendroid.

Given a continuum $X$, we consider the following hyperspaces of $X$ :

$$
2^{X}=\{A \subset X \mid A \text { is nonempty and closed }\}
$$

and

$$
\mathcal{C}_{n}(X)=\left\{A \in 2^{X} \mid A \text { has at most } n \text { components }\right\},
$$

where $n$ is a positive integer. $\mathcal{C}_{n}(X)$ is called the $n$-fold hyperspace of $X$. These spaces are topologized with the Hausdorff metric defined as follows:

$$
\mathcal{H}(A, B)=\inf \left\{\varepsilon>0 \mid A \subset \mathcal{V}_{\varepsilon}(B) \text { and } B \subset \mathcal{V}_{\varepsilon}(A)\right\},
$$

$\mathcal{H}$ always denotes the Hausdorff metric on $2^{X}$. When $n=1$, we write $\mathcal{C}(X)$ instead of $\mathcal{C}_{1}(X)$.

The symbol $\mathcal{F}_{n}(X)$ denotes the $n$-fold symmetric product of $X$; that is:

$$
\mathcal{F}_{n}(X)=\left\{A \in \mathcal{C}_{n}(X) \mid A \text { has at most } n \text { points }\right\} .
$$

Note that, by definition, $\mathcal{F}_{n}(X) \subset \mathcal{C}_{n}(X)$. It is known that if $X$ is a continuum, then $2^{X}$ and $\mathcal{C}_{n}(X)$ are arcwise connected continua (for $2^{X}$ and $\mathcal{C}(X)$ see $[29,(1.13)]$; for $\mathcal{C}_{n}(X)$ and $n \geq 2$, see [24, 3.1]). Also, $\mathcal{F}_{n}(X)$ is a continuum for all positive integers $n([4$, p. 877]).

A continuum $X$ has the property of Kelley provided that for each $\varepsilon>0$, there exists $\delta>0$ such that for every pair of points $x$ and $y$ of $X$ such that $d(x, y)<\delta$ and each subcontinuum $A$ of $X$ such that $x \in A$, there exists a subcontinuum $B$ of $X$ such that $y \in B$ and $\mathcal{H}(A, B)<\varepsilon$.

If $A$ is a nonempty subset of $X, \mathcal{C}_{n}(A)$ denotes the set $\left\{B \in \mathcal{C}_{n}(X) \mid B \subset\right.$ $A\}$.

\section{Preliminary Results}

The following theorem is due to David P. Bellamy and it is part of [1, Example II].

THEOREM 3.1. If $X$ is a continuum which is the continuous image of the Cantor fan, then $X$ is arcwise decomposable.

Proof. Let $\mathfrak{F}_{\mathfrak{C}}$ be the Cantor fan, and let $f: \mathfrak{F}_{\mathfrak{C}} \rightarrow X$ be a surjective map. By the Kuratowski-Zorn lemma ([19, Theorem 25, p. 33]), there exists a subcontinuum $K$ of $\mathfrak{F}_{\mathfrak{C}}$ such that $f(K)=X$ and $f(L) \neq X$ for any proper subcontinuum $L$ of $K$. If the vertex $\nu$ of $\mathfrak{F}_{\mathfrak{C}}$ is such that $K \backslash\{\nu\}$ is not connected, then $K$ is the union of two arcwise connected proper subcontinua. If $K \backslash\{\nu\}$ is connected, then $K$ is an arc. In either case, there exist two arcwise connected proper subcontinua $K_{1}$ and $K_{2}$ of $K$ such that $K=K_{1} \cup K_{2}$. Hence, by the minimality of $K, f\left(K_{1}\right)$ and $f\left(K_{2}\right)$ are arcwise connected proper subcontinua of $X$ such that $X=f\left(K_{1}\right) \cup f\left(K_{2}\right)$. Therefore, $X$ is arcwise decomposable. 
The following result is known, but we could not find a proof of it.

THEOREM 3.2. If $X$ is a continuum with trivial shape and $Y$ is a subcontinuum of $X$ which is a retract of $X$, then $Y$ has trivial shape.

Proof. Let $r: X \rightarrow Y$ be a retraction. Let $Z$ be an absolute neighborhood retract and let $f: Y \rightarrow Z$ be a map. Since $X$ has trivial shape, the map $f \circ r: X \rightarrow Z$ is homotopic to a constant map. Let $H: X \times[0,1] \rightarrow Z$ be a homotopy such that $H((x, 0))=f \circ r(x)$ and $H((x, 1))=z_{0}$ for all $x \in X$ and some $z_{0} \in Z$. Let $G=\left.H\right|_{Y \times[0,1]}$. Then $G: Y \times[0,1] \rightarrow Z$ is a homotopy such that $G((y, 0))=f \circ r(y)=f(y)$ and $H((y, 1))=z_{0}$ for each $y \in Y$. Hence, $f$ is homotopic to a constant map. Therefore, $Y$ has trivial shape.

From the proof of $[17,1.1]$, we obtain the following lemma. It is worth mentioning that this result does not extend to $n$-fold symmetric products for $n \geq 3$. It is known that $\mathcal{F}_{3}\left(\mathcal{S}^{1}\right)$ is homeomorphic to the 3 -sphere $\mathcal{S}^{3}([5])$ and any map from $\mathcal{S}^{3}$ into $\mathcal{S}^{1}$ is inessential ([11, p. 343]).

LEMMA 3.3. Let $X$ be a continuum such that there exists an essential map from $X$ onto $\mathcal{S}^{1}$. Then there exists an essential map from $\mathcal{F}_{2}(X)$ onto $\mathcal{S}^{1}$.

THEOREM 3.4. If $X$ is a proper circle-like continuum, then $\mathcal{F}_{2}(X)$ does not have trivial shape.

Proof. By [20, 3.1], there exists an essential map from $X$ onto $\mathcal{S}^{1}$. Then, by Lemma 3.3, there exists an essential map from $\mathcal{F}_{2}(X)$ onto $\mathcal{S}^{1}$. Therefore, $\mathcal{F}_{2}(X)$ does not have trivial shape.

QUESTION 3.1. If $X$ is a proper circle-like continuum and $n \geq 3$, then is it true that $\mathcal{F}_{n}(X)$ does not have trivial shape?

Recall that a convex metric $\rho$ on a continuum $X$ is a metric which satisfies that for every two points $x$ and $y$ in $X$, there exists a point $z$ in $X$ such that $\rho(x, z)=\rho(y, z)=\frac{1}{2} \rho(x, y)$. If $X$ is a continuum with a convex metric $\rho$ and $n$ is a positive integer, we define the following two functions:

$$
K_{\rho}:[0, \infty) \times 2^{X} \rightarrow 2^{X}
$$

and

$$
\alpha_{\rho}^{n}: 2^{X} \rightarrow \mathbb{R}
$$

by

$$
K_{\rho}((t, A))=\{x \in X \mid \rho(x, a) \leq t \text { for some } a \in A\}
$$

and

$$
\alpha_{\rho}^{n}(A)=\inf \left\{t \geq 0 \mid K_{\rho}((t, A)) \in \mathcal{C}_{n}(X)\right\} .
$$

LEMma 3.5. Let $X$ be a locally connected continuum with a convex metric $\rho$ and let $n$ and $m$ be positive integers such that $m \geq n$. Then 
(1) If $A \in \mathcal{C}_{m}(X)$ and $s \in[0,1]$, then $\alpha_{\rho}^{n}\left(K_{\rho}\left(\left(s \cdot \alpha_{\rho}^{n}(A), A\right)\right)\right)=(1-s)$. $\alpha_{\rho}^{n}(A)$.

(2) Let $R: \mathcal{C}_{m}(X) \rightarrow \mathcal{C}_{n}(X)$ be given by $R(A)=K_{\rho}\left(\left(\alpha_{\rho}^{n}(A), A\right)\right.$ ). If $A \in$ $R^{-1}(B)$ and $s \in[0,1]$, then $K_{\rho}\left(\left(s \cdot \alpha_{\rho}^{n}(A), A\right)\right) \in R^{-1}(B)$.

Proof. We show (1). Note that, by $[29,(0.65 .3)(\mathrm{c})]$, we have that:

$$
\begin{aligned}
K_{\rho}(((1-s) & \left.\left.\cdot \alpha_{\rho}^{n}(A), K_{\rho}\left(\left(s \cdot \alpha_{\rho}^{n}(A), A\right)\right)\right)\right) \\
& =K_{\rho}\left(\left((1-s) \cdot \alpha_{\rho}^{n}(A)+s \cdot \alpha_{\rho}^{n}(A), A\right)\right) \\
& =K_{\rho}\left(\left(\alpha_{\rho}^{n}(A), A\right)\right) .
\end{aligned}
$$

Now, it follows that $\alpha_{\rho}^{n}\left(K_{\rho}\left(\left(s \cdot \alpha_{\rho}^{n}(A), A\right)\right)\right)=(1-s) \cdot \alpha_{\rho}^{n}(A)$.

To prove (2), let $B \in \mathcal{C}_{n}(X)$, let $A \in R^{-1}(B)$ and let $s \in[0,1]$. Then it follows from the definition of $R,(1)$ and $[29,(0.65 .3)$ (c)], that

$$
\begin{aligned}
R\left(K_{\rho}\left(\left(s \cdot \alpha_{\rho}^{n}(A), A\right)\right)\right) & =K_{\rho}\left(\left(\alpha_{\rho}^{n}\left(K_{\rho}\left(\left(s \cdot \alpha_{\rho}^{n}(A), A\right)\right)\right), K_{\rho}\left(\left(s \cdot \alpha_{\rho}^{n}(A), A\right)\right)\right)\right) \\
& =K_{\rho}\left(\left((1-s) \cdot \alpha_{\rho}^{n}(A), K_{\rho}\left(\left(s \cdot \alpha_{\rho}^{n}(A), A\right)\right)\right)\right) \\
& =K_{\rho}\left(\left(\alpha_{\rho}^{n}(A), A\right)\right)=B .
\end{aligned}
$$

\section{RETRACTS}

TheOREM 4.1. Let $X$ be a continuum and let $n$ and $m$ be positive integers such that $m \geq n$. If $\mathcal{F}_{n}(X)$ is a retract of $\mathcal{C}_{m}(X)$, then:

(1) $X$ is arcwise connected;

(2) $\mathcal{F}_{n}(X)$ is arcwise decomposable:

(3) $\mathcal{F}_{n}(X)$ is uniformly pathwise connected:

(4) $\mathcal{F}_{n}(X)$ is weakly chainable;

(5) $\mathcal{F}_{n}(X)$ has trivial shape;

(6) $\mathcal{F}_{n}(X)$ is movable.

Proof. Suppose $\mathcal{F}_{n}(X)$ is a retract of $\mathcal{C}_{m}(X)$. Since $n$-fold hyperspaces are arcwise connected continua (see $[24,3.1]$ or $[26,1.8 .12]$ ), we have that $\mathcal{F}_{n}(X)$ is arcwise connected. Thus, $X$ is arcwise connected $([10,2.7])$. By $[18$, Theorem 2.3 and the Remark on p. 29], there exists a map from the Cantor fan $\mathfrak{F}_{\mathfrak{C}}$ onto $\mathcal{C}_{m}(X)$. Hence, there exists a map from $\mathfrak{F}_{\mathfrak{C}}$ onto $\mathcal{F}_{n}(X)$. This implies that $\mathcal{F}_{n}(X)$ is arcwise decomposable, by Theorem 3.1, and uniformly pathwise connected $([22,3.5])$. By $[10,3.3], \mathcal{C}_{m}(X)$ is weakly chainable. Thus, $\mathcal{F}_{n}(X)$ is weakly chainable. Since $\mathcal{C}_{m}(X)$ has trivial shape $([24,4.6]$ or $[26,6.2 .2])$, by Theorem 3.2, $\mathcal{F}_{n}(X)$ has trivial shape. Since $\mathcal{F}_{n}(X)$ has trivial shape, $\mathcal{F}_{n}(X)$ is movable $([3,(3.2)])$.

Recall that if $\mathcal{S}^{1}$ is the unit circle, then $\mathcal{F}_{2}\left(\mathcal{S}^{1}\right)$ is homeomorphic to the Möbius strip ([4, p. 877]), which is not unicoherent. Note the following: 
Corollary 4.2. Let $X$ be a continuum and let $m \geq 2$ be an integer. If $\mathcal{F}_{2}(X)$ is a retract of $\mathcal{C}_{m}(X)$, then $\mathcal{F}_{2}(X)$ is unicoherent.

Proof. By Theorem 4.1, $\mathcal{F}_{2}(X)$ has trivial shape. Hence, by $[21,2.1]$, $\mathcal{F}_{2}(X)$ is contractible with respect to any absolute neighborhood retract. In particular, $\mathcal{F}_{2}(X)$ is contractible with respect to $\mathcal{S}^{1}$. Hence, by [23, Theorem 1, p. 434], $\mathcal{F}_{2}(X)$ is unicoherent.

Corollary 4.3. An arc-like continuum $X$ is an arc if and only if $\mathcal{F}_{n}(X)$ is a retract of $\mathcal{C}_{m}(X)$ for some positive integers $n$ and $m$.

Proof. Let $X$ be an arc-like continuum such that there $\mathcal{F}_{n}(X)$ is a retract of $\mathcal{C}_{m}(X)$ for some positive integers $m$ and $n$ such that $m \geq n$. By Theorem 4.1, $X$ is arcwise connected. Since the arc is the only arcwise connected arc-like continuum, we have that $X$ is an arc.

The reverse implication follows from Corollary 4.10 .

Corollary 4.4. If $X$ is a circle-like continuum and $m \geq 2$ is an integer, then there does not exist a retraction from $\mathcal{C}_{m}(X)$ onto $\mathcal{F}_{2}(X)$.

Proof. If $X$ is also an arc-like continuum, it follows from [6, Theorem 3] that $X$ is either indecomposable or the union of two indecomposable continua. Hence, by Corollary 4.3, there does not exist a retraction from $\mathcal{C}_{m}(X)$ onto $\mathcal{F}_{2}(X)$. Suppose $X$ is a proper circle-like continuum. Then, by Theorem 3.4, $\mathcal{F}_{2}(X)$ does not have trivial shape. Therefore, by Theorem 4.1, there does not exist a retraction from $\mathcal{C}_{m}(X)$ onto $\mathcal{F}_{2}(X)$.

THEOREM 4.5. If $X$ is a $k$-dimensional continuum containing a $k$-sphere and $n$ and $m$ are positive integers such that $m \geq n$, then there does not exist a retraction from $\mathcal{C}_{m}(X)$ onto $\mathcal{F}_{n}(X)$.

Proof. By definition, a $k$-sphere does not have trivial shape. Since $X$ is a $k$-dimensional continuum containing a $k$-sphere $S$, there exists a retraction from $X$ onto $S$ ([16, Theorem VI 4, p. 83]). Hence, by Lemma 3.2, $X$ does not have trivial shape. Therefore, by Theorem 4.1 , there does not exist a retraction from $\mathcal{C}_{m}(X)$ onto $\mathcal{F}_{n}(X)$.

In $[8,3.1]$ it is shown that if $X$ is a one-dimensional continuum and $\mathcal{F}_{1}(X)$ is a retract of $\mathcal{C}(X)$, then $X$ is a dendroid. Related to this, we have the following:

Corollary 4.6. Let $X$ be a one-dimensional continuum and let $n$ and $m$ be positive integers such that $m \geq n$. If $\mathcal{F}_{n}(X)$ is a retract of $\mathcal{C}_{m}(X)$, then $X$ is uniquely arcwise connected.

The following theorem extends $[8,3.1]$ to $n$-fold hyperspaces. 
Theorem 4.7. Let $X$ be a one-dimensional continuum and let $n$ be an integer. If $\mathcal{F}_{1}(X)$ is a retract of $\mathcal{C}_{n}(X)$, then $X$ is a uniformly pathwise connected dendroid.

Proof. Since $\mathcal{F}_{1}(X)$ is homeomorphic to $X$, by Theorem 4.1, $X$ is a uniformly pathwise connected continuum with trivial shape. By $[21,2.1$ (B)], $X$ is tree-like. Since tree-like continua are hereditarily unicoherent ([7, Theorem 1]), we have that $X$ is a dendroid.

Theorem 4.8. Let $X$ be a continuum, let $Y$ be a subcontinuum of $X$ which is a retract of $X$, and let $n$ and $m$ be positive integers such that $m \geq n$. If $\mathcal{F}_{n}(X)$ is a retract (a deformation retract or a strong deformation retract, respectively) of $\mathcal{C}_{m}(X)$, then $\mathcal{F}_{n}(Y)$ is a retract (a deformation retract or a strong deformation retract, respectively) of $\mathcal{C}_{m}(Y)$.

Proof. Let $r: X \rightarrow Y$ be a retraction. Then $\mathcal{C}_{m}(r): \mathcal{C}_{m}(X) \rightarrow \mathcal{C}_{m}(Y)$ is a retraction.

Suppose $\mathcal{F}_{n}(X)$ is a retract of $\mathcal{C}_{m}(X)$. Let $R: \mathcal{C}_{m}(X) \rightarrow \mathcal{F}_{n}(X)$ be a retraction. Define $L: \mathcal{C}_{m}(Y) \rightarrow \mathcal{F}_{n}(Y)$ by $L(A)=\mathcal{C}_{m}(r)(R(A))$. Then $L$ is well defined and continuous. If $A \in \mathcal{F}_{n}(Y)$, then $L(A)=\mathcal{C}_{m}(r)(R(A))=$ $\mathcal{C}_{m}(r)(A)=A$. Therefore, $L$ is a retraction.

Now, suppose that $\mathcal{F}_{n}(X)$ is a (strong) deformation retract of $\mathcal{C}_{m}(X)$. Let $H: \mathcal{C}_{m}(X) \times[0,1] \rightarrow \mathcal{C}_{m}(X)$ be a map such that $H((A, 0))=A$ and $H((A, 1))=R(A)$, where $R: \mathcal{C}_{m}(X) \rightarrow \mathcal{F}_{n}(X)$ is a retraction $($ and $H((A, t))=$ $A$ for all $A \in \mathcal{F}_{n}(X)$ and all $\left.t \in[0,1]\right)$. Define $G: \mathcal{C}_{m}(Y) \times[0,1] \rightarrow \mathcal{C}_{m}(Y)$ by $G((A, t))=\mathcal{C}_{m}(r)(H((A, t)))$. Then $G$ is well defined and continuous. Also, we have that $G((A, 0))=\mathcal{C}_{m}(r)(H((A, 0)))=\mathcal{C}_{m}(r)(A)=A$ and $G((A, 1))=\mathcal{C}_{m}(r)(H((A, 1)))=\mathcal{C}_{m}(r)(R(A)) \in \mathcal{F}_{n}(Y)$ (and $G((A, t))=$ $\mathcal{C}_{m}(r)(H((A, t)))=\mathcal{C}_{m}(r)(A)=A$ for all $A \in \mathcal{F}_{n}(Y)$ and all $\left.t \in[0,1]\right)$. Therefore, $\mathcal{F}_{n}(Y)$ is a (strong) deformation retract of $\mathcal{C}_{m}(Y)$.

We continue noting that $[27,3.2]$ can be strengthened as follows:

TheOrem 4.9. Let $X$ be a locally connected continuum and let $n$ and $m$ be positive integers such that $m \geq n$. If $\mathcal{F}_{n}(X)$ is an absolute retract, then $\mathcal{F}_{n}(X)$ is a strong deformation retract of $\mathcal{C}_{m}(X)$.

Proof. Observe that in the proof of [27, 3.1], it is only used the fact that $\mathcal{F}_{n}(X)$ is an absolute retract. Hence, we conclude that there exists a strong deformation retraction $H: 2^{X} \times[0,1] \rightarrow 2^{X}$ from $2^{X}$ onto $\mathcal{F}_{n}(X)$.

Since $X$ is locally connected, $\mathcal{C}_{m}(X)$ is an absolute retract ([32, Théorème $\left.\left.\mathrm{II}_{m}\right]\right)$. Hence, there exists a retraction $r: 2^{X} \rightarrow \mathcal{C}_{m}(X)$. Thus, the map $r \circ$ $\left(\left.H\right|_{\mathcal{C}_{m}(X) \times[0,1]}\right): \mathcal{C}_{m}(X) \times[0,1] \rightarrow \mathcal{C}_{m}(X)$ is a strong deformation retraction from $\mathcal{C}_{m}(X)$ onto $\mathcal{F}_{n}(X)$.

As a consequence of Theorem 4.9 and the fact that the $n$-fold symmetric product of an absolute retract is an absolute retract ([12, p. 316]), we have: 
COROLlary 4.10. If $X$ is an absolute retract and $n$ and $m$ are positive integers such that $m \geq n$, then $\mathcal{F}_{n}(X)$ is a strong deformation retract of $\mathcal{C}_{m}(X)$.

Since dendrites are absolute retracts ([23, Theorem 16, p. 344]), we obtain:

COROLlary 4.11. If $X$ is a dendrite and $n$ and $m$ are positive integers such that $m \geq n$, then $\mathcal{F}_{n}(X)$ is a strong deformation retract of $\mathcal{C}_{m}(X)$.

THEOREM 4.12. Let $X$ be a locally connected continuum and let $n$ and $m$ be positive integers such that $m \geq n$. Then $\mathcal{F}_{n}(X)$ is a retract of $\mathcal{C}_{m}(X)$ if and only if $\mathcal{F}_{n}(X)$ is an absolute retract.

Proof. If $\mathcal{F}_{n}(X)$ is an absolute retract, then, clearly, $\mathcal{F}_{n}(X)$ is a retract of $\mathcal{C}_{m}(X)$. Suppose $\mathcal{F}_{n}(X)$ is a retract of $\mathcal{C}_{m}(X)$. Since $X$ is locally connected, $\mathcal{C}_{m}(X)$ is an absolute retract $\left(\left[32\right.\right.$, Théorème $\left.\left.\mathrm{II}_{m}\right]\right)$. Hence, $\mathcal{F}_{n}(X)$ is an absolute retract $([23$, Theorem 6, p. 341]).

The next result strengthens [25, 4.13] (see also [26, 6.7.17]).

Corollary 4.13. Let $X$ be a locally connected continuum and let $n$ and $m$ be positive integers such that $m \geq n$. Then the following are equivalent:

(1) $\mathcal{F}_{n}(X)$ is an absolute retract;

(2) $\mathcal{F}_{n}(X)$ is a retract of $\mathcal{C}_{m}(X)$;

(3) $\mathcal{F}_{n}(X)$ is a deformation retract of $\mathcal{C}_{m}(X)$;

(4) $\mathcal{F}_{n}(X)$ is a strong deformation retract of $\mathcal{C}_{m}(X)$.

Proof. Clearly, (4) implies (3) and (3) implies (2). By Theorem 4.12, (2) implies (1). By Theorem 4.9, (1) implies (4).

THEOREM 4.14. Let $X$ be a continuum and let $n$ and $m$ be positive integers such that $m \geq n$. If $\mathcal{F}_{n}(X)$ is a deformation retract (or a strong deformation retract) of $2^{X}$, then $\mathcal{F}_{n}(X)$ is a deformation retract (or a strong deformation retract) of $\mathcal{C}_{m}(X)$.

Proof. Let $H: 2^{X} \times[0,1] \rightarrow 2^{X}$ be a deformation retraction from $2^{X}$ onto $\mathcal{F}_{n}(X)$. Let $R: \mathcal{C}_{m}(X) \rightarrow \mathcal{F}_{n}(X)$ be given by $R(A)=H((A, 1))$. Define $G: \mathcal{C}_{m}(X) \times[0,1] \rightarrow \mathcal{C}_{m}(X)$ by

$$
G((A, t))= \begin{cases}\cup\{H((A, s)) \mid 0 \leq s \leq 2 t\}, & \text { if } t \in\left[0, \frac{1}{2}\right] \\ \cup\{H((A, s)) \mid 2 t-1 \leq s \leq 1\}, & \text { if } t \in\left[\frac{1}{2}, 1\right]\end{cases}
$$

Note that if $t=\frac{1}{2}$, then both definitions of $G$ give the value $\cup\{H((A, s)) \mid 0 \leq$ $s \leq 1\}$. Let $t \in\left[0, \frac{1}{2}\right]$. Since $H$ is continuous, $\{H((A, s)) \mid 0 \leq s \leq 2 t\}$ is a closed subset of $2^{X}$ that contains the element $H((A, 0))=A$, and $A \in \mathcal{C}_{m}(X)$. In fact, $\left.G\right|_{\mathcal{C}_{m}(X) \times\left[0, \frac{1}{2}\right]}$ is a segment homotopy $([29,(16.4)])$. Hence, $G((A, t)) \in$ $\mathcal{C}_{m}(X)$ for each $(A, t) \in \mathcal{C}_{m}(X) \times\left[0, \frac{1}{2}\right]([9$, Proposition 3$])$. Now let $t \in\left[\frac{1}{2}, 1\right]$. By the continuity of $H,\{H((A, s)) \mid 2 t-1 \leq s \leq 1\}$ is a closed subset of 
$2^{X}$ that contains the element $H((A, 1)) \in \mathcal{F}_{n}(X)$. Again, $\left.G\right|_{\mathcal{C}_{m}(X) \times\left[\frac{1}{2}, 1\right]}$ is a segment homotopy, in reverse order, from $G\left(\left(A, \frac{1}{2}\right)\right) \in \mathcal{C}_{m}(X)$ to $G((A, 1))=$ $H((A, 1)) \in \mathcal{F}_{n}(X)$. Thus, $G((A, t)) \in \mathcal{C}_{m}(X)$ for each $(A, t) \in \mathcal{C}_{m}(X) \times\left[\frac{1}{2}, 1\right]$ ( $[9$, Proposition 3]). Hence, $G$ is well defined. Since $H$ and the union map ([29, (1.48)]) are continuous, it follows that $G$ is continuous. Note that $G((A, 0))=$ $H((A, 0))=A$ and $G((A, 1))=H((A, 1))=R(A) \in \mathcal{F}_{n}(X)$. Therefore, $\mathcal{F}_{n}(X)$ is a deformation retract of $\mathcal{C}_{m}(X)$.

Let $H: 2^{X} \times[0,1] \rightarrow 2^{X}$ be a strong deformation retraction from $2^{X}$ onto $\mathcal{F}_{n}(X)$. If $G$ is defined as in the previous paragraph, then $G((A, t))=A$ for each $A \in \mathcal{F}_{n}(X)$ and each $t \in[0,1]$. Hence, $G$ is a strong deformation retraction. Therefore, $\mathcal{F}_{n}(X)$ is a strong deformation retract of $\mathcal{C}_{m}(X)$.

The next theorem strengthens [25, 4.14 and 4.15] (see also [26, 6.7.18]), with some changes in the proof, we include the details for the convenience of the reader.

TheOREM 4.15. Let $X$ be a locally connected continuum and let $n$ and $m$ be positive integers such that $m \geq n$. Then the following hold:

(1) $\mathcal{C}_{n}(X)$ is a retract of $\mathcal{C}_{m}(X)$

(2) $\mathcal{C}_{n}(X)$ is a deformation retract of $\mathcal{C}_{m}(X)$;

(3) $\mathcal{C}_{n}(X)$ is a strong deformation retract of $\mathcal{C}_{m}(X)$.

Proof. Since $X$ is locally connected, by $\left[32\right.$, Théorème $\left.\mathrm{II}_{m}\right], \mathcal{C}_{n}(X)$ is an absolute retract. Hence, (1) holds. Next, note that, since $X$ is locally connected, then $X$ has the property of Kelley $([29,(16.11)])$. Hence, $\mathcal{C}_{m}(X)$ is contractible $([24,3.8])$. Thus, by [31, 32E.4], (1) and (2) are equivalent. By $[26,6.7 .14],(3)$ is equivalent to the fact that $X$ is locally connected.

The next result extends $[29,(6.12)]$ to $n$-fold hyprespaces. We include the details of the proof in order to present the appropriate changes needed.

THEOREM 4.16. Let $X$ be a continuum and let $n$ and $m$ be positive integers such that $m \geq n+1$. Then there exists a retraction $R: \mathcal{C}_{m}(X) \rightarrow \mathcal{C}_{n}(X)$ such that $A \subset R(A)$ for each $A \in \mathcal{C}_{m}(X)$ if and only if $X$ is locally connected. Furthermore, if $X$ is locally connected, then there exists a retraction $R: \mathcal{C}_{m}(X) \rightarrow \mathcal{C}_{n}(X)$ such that $A \subset R(A)$ for each $A \in \mathcal{C}_{m}(X)$, such that $R$ is monotone and, moreover, $R^{-1}(B)$ is contractible for every $B \in \mathcal{C}_{n}(X)$.

Proof. Suppose $R: \mathcal{C}_{m}(X) \rightarrow \mathcal{C}_{n}(X)$ is a retraction such that $A \subset R(A)$ for all $A \in \mathcal{C}_{m}(X)$. Suppose $X$ is not locally connected. Then there exists a point $p$ of $X$ such that $X$ is not connected im kleinen at $p$. Hence, there exist $\varepsilon>0$ and a sequence $\left\{K_{\ell}\right\}_{\ell=1}^{\infty}$ of components of $C l\left(\mathcal{V}_{\varepsilon}(p)\right)$ converging to a continuum $K \subset C l\left(\mathcal{V}_{\varepsilon}(p)\right)$ such that $p \in K$ and $K \cap K_{\ell}=\emptyset$ for each positive integer $\ell\left(\left[30,(12.1)\right.\right.$, p. 18]). Let $\left\{p_{\ell}\right\}_{\ell=1}^{\infty}$ be a sequence of points of $C l\left(\mathcal{V}_{\varepsilon}(p)\right)$ converging to $p$ such that $p_{\ell} \in K_{\ell}$ for every positive integer $\ell$. Given a positive 
integer $j$, let $A_{j}=\{p\} \cup\left\{p_{j+\ell}\right\}_{\ell=1}^{m-1}$. Observe that $A_{j} \cap K \neq \emptyset$ and $A_{j} \cap K_{j+\ell} \neq$ $\emptyset$ for each positive integer $j$ and every $\ell \in\{1, \ldots, m-1\}$. By hypothesis, each $A_{j}$ is a subset of $R\left(A_{j}\right)$. Hence, $R\left(A_{j}\right) \cap K \neq \emptyset$ and $R\left(A_{j}\right) \cap K_{j+\ell} \neq \emptyset$ for each positive integer $j$ and every $\ell \in\{1, \ldots, m-1\}$. Let $j$ be a positive integer. Since $R\left(A_{j}\right)$ has at most $n$ components, $m \geq n+1$ and must intersect $m$ pairwise disjoint subsets of $C l\left(\mathcal{V}_{\varepsilon}(p)\right)$, there exists a component $B_{j}$ of $R\left(A_{j}\right)$ such that $B_{j} \cap\left(X \backslash C l\left(\mathcal{V}_{\varepsilon}(p)\right)\right) \neq \emptyset$. Thus, $\operatorname{diam}\left(R\left(A_{j}\right)\right) \geq \varepsilon$. Note that, by construction, the sequence $\left\{A_{j}\right\}_{j=1}^{\infty}$ converges to $\{p\}$. Hence, by the continuity of $R$, the sequence $\left\{R\left(A_{j}\right)\right\}_{j=1}^{\infty}$ converges to $R(\{p\})$. Since $R$ is a retraction, and $\{p\} \in \mathcal{C}_{n}(X), R(\{p\})=\{p\}$. Hence, the sequence $\left\{R\left(A_{j}\right)\right\}_{j=1}^{\infty}$ converges to $\{p\}$. A contradiction to the fact that $\operatorname{diam}\left(R\left(A_{j}\right)\right) \geq \varepsilon$ for all positive integers $j$. Therefore, $X$ is locally connected.

Next, assume that $X$ is locally connected with a convex metric $\rho$ ([2] or [28]). Let $R: \mathcal{C}_{m}(X) \rightarrow \mathcal{C}_{n}(X)$ be given by $R(A)=K_{\rho}\left(\left(\alpha_{\rho}^{n}(A), A\right)\right)$. By $[25,4.9]$ or $[26,6.7 .13], R$ is well defined and continuous. It is easy to prove that $R$ is a retraction. Let $B \in \mathcal{C}_{n}(X)$. For each $(A, s) \in R^{-1}(B) \times[0,1]$, let $h: R^{-1}(B) \times[0,1] \rightarrow R^{-1}(B)$ be given by $h((A, s))=K_{\rho}\left(\left(s \cdot \alpha_{\rho}^{n}(A), A\right)\right)$. By (2) of Lemma 3.5, $h$ is well defined. Since $K_{\rho}$ and $\alpha_{\rho}^{n}$ are continuous $([29,(0.65 .3)(\mathrm{f})]$ and $[25,4.9]$, respectively), $h$ is continuous. Note that $h((A, 0))=K_{\rho}((0, A))=A$ and $h((A, 1))=K_{\rho}\left(\left(\alpha_{\rho}^{n}(A), A\right)\right)=R(A)=B$ for all $A \in R^{-1}(B)$. Therefore, $R^{-1}(B)$ is contractible.

ACKNOWLEDGEMENTS.

The author thanks the referee for the valuable suggestions which improved the paper.

\section{REFERENCES}

[1] D. P. Bellamy, The cone over the Cantor set-continuous maps from both directions, in: Proceedings of the Topology Conference, Emory University, Atlanta, 1970, 8-25.

[2] R. H. Bing, Partitioning a set, Bull. Amer. Math. Soc. 55 (1949), 1101-1110.

[3] K. Borsuk, Some remarks on shape properties of compacta, Fund. Math. 85 (1974), $185-195$.

[4] K. Borsuk and S. Ulam, On symmetric products of topological spaces, Bull. Amer. Math. Soc. 37 (1931), 875-882.

[5] R. Bott, On the third symmetric potency of $\mathcal{S}_{1}$, Fund. Math. 39 (1952), 264-268.

[6] C. E. Burgess, Chainable continua and indecomposability, Pacific J. Math. 9 (1959), 653-659.

[7] J. H. Case and R. E. Chamberlin, Characterizations of tree-like continua, Pacific J. Math. 10 (1960), 73-84.

[8] J. J. Charatonik, W. J. Charatonik, K. Omiljanowski and J. R. Prajs, Hyperspace retractions for curves, Dissertationes Math. (Rozprawy Mat.) 370 (1997), 1-34.

[9] J. J. Charatonik, A. Illanes and S. Macías, Induced mappings on the hyperspace $\mathcal{C}_{n}(X)$ of a continuum X, Houston J. Math. 28 (2002), 781-805.

[10] J. J. Charatonik and S. Macías, Mappings of some hyperspaces, JP J. Geom. Topol. 4 (2004), 53-80.

[11] J. Dugundji, Topology, Allyn and Bacon, Inc., Boston, 1966.

[12] T. Ganea, Symmetrische Potenzen topologischer Räume, Math. Nachr. 11 (1954), 305-316. 
[13] J. T. Goodykoontz, $\mathcal{C}(X)$ is not necessarily a retract of $2^{X}$, Proc. Amer. Math. Soc. 67 (1977), 177-178.

[14] J. T. Goodykoontz, A non-locally connected continuum $X$ such that $\mathcal{C}(X)$ is a retract of $2^{X}$, Proc. Amer. Math. Soc. 91 (1984), 379-322.

[15] J. T. Goodykoontz, Some retractions and deformation retractions on $2^{X}$ and $\mathcal{C}(X)$, Topology Appl. 21 (1985), 121-133.

[16] W. Hurewicz and H. Wallman, Dimension theory, Princeton Univ. Press, Princeton, 1948.

[17] A. Illanes, Multicoherence of symmetric products, An. Inst. Mat. Univ. Nac. Autónoma México 25 (1985), 11-24.

[18] J. L. Kelley, Hyperspaces of a continuum, Trans. Amer. Math. Soc. 52 (1942), 22-36.

[19] J. L. Kelley, General topology, Graduate Texts in Mathematics 27, Springer-Verlag, New York-Berlin, 1975, reprint of the 1955 ed. published by Van Nostrand.

[20] J. Krasinkiewicz, On the hyperspaces of snake-like and circle-like continua, Fund. Math. 84 (1974), 155-164.

[21] J. Krasinkiewicz, Curves which are continuous images of tree-like continua are movable, Fund. Math. 89 (1975), 233-260.

[22] W. Kuperberg, Uniformly pathwise connected continua, in: Studies in topology, Proc. Conf. Univ. North Carolina, Charlotte, NC, 1974, Academic Press, New York, 1975, $315-324$.

[23] K. Kuratowski, Topology, Vol. 2, English transl., Academic Press, New York-London, PWN, Warsaw, 1968.

[24] S. Macías, On the hyperspaces $\mathcal{C}_{n}(X)$ of a continuum X, Topology Appl. 109 (2001), $237-256$.

[25] S. Macías, On the hyperspaces $\mathcal{C}_{n}(X)$ of a continuum X, II, Topology Proc. 25 (2000), 255-276.

[26] S. Macías, Topics on continua, Chapman \& Hall/CRC, Boca Raton, 2005.

[27] S. Macías, On the n-fold hyperspace suspension of continua, II, Glas. Mat. Ser. III 41(61) (2006), 335-343.

[28] E. E. Moise, Grille decomposition and convexification theorem for compact metric locally connected continua, Bull. Amer. Math. Soc. 55 (1949), 1111-1121.

[29] S. B. Nadler, Jr., Hyperspaces of sets. A text with research questions, Monographs and Textbooks in Pure and Applied Math. 49, Marcel Dekker, New York-Basel, 1978. Reprinted in: Aportaciones Matemáticas de la Sociedad Matemática Mexicana, Serie Textos \# 33, 2006.

[30] G. T. Whyburn, Analytic topology, American Mathematical Society Colloquium Publications 28, AMS, Providence, 1942, reprinted with corrections 1971.

[31] S. Willard, General topology, Addison-Wesley Publishing Co., Reading, Mass.London-Don Mills, 1970.

[32] M. Wojdisławski, Rétractes absolus et hyperespaces des continus, Fund. Math. 32 (1939), 184-192.

S. Macías

Instituto de Matemáticas

Universidad Nacional Autónoma de México

Circuito Exterior, Ciudad Universitaria, México D. F., C. P. 04510

México

E-mail: macias@servidor.unam.mx

Received: 12.4.2010.

Revised: 29.7.2010. \& 5.8.2010. 